\title{
Association and Interaction Effect between VEGF Receptor-2 (VEGFR-2) Gene Polymorphisms and Dietary Pattern on Blood Lipids in Malaysian Adults
}

\author{
Roseline Wai Kuan Yap ${ }^{1, *}$, Motofumi Masaki ${ }^{2}$, Yoshihiro Shidoji ${ }^{2}$ and Wei Min Hon ${ }^{3}$ \\ ${ }^{1}$ School of Biosciences, Taylor's University, Selangor, Malaysia \\ ${ }^{2}$ Graduate School of Human Health Science, University of Nagasaki, Nagayo, Nagasaki, Japan \\ ${ }^{3}$ Vice Chancellery, KDU University College, Selangor, Malaysia
}

\begin{abstract}
Background: Vascular endothelial growth factor receptor-2 (VEGFR-2) and diet are related to blood lipid health. Gene-diet interaction studies can identify the risks. The objectives are to determine associations, and interaction effects between VEGFR-2 gene polymorphisms (rs1870377 and rs2071559) and dietary patterns on blood lipids in multiethnic Malaysian adults.

Methods: Dietary intakes of 509 (153 Malay, 179 Chinese, and 177 Indian) Malaysians were obtained from food frequency questionnaire for the construction of dietary patterns using factor analysis. Anthropometric measurements: body mass index and blood pressure; and biomarkers: glycated hemoglobin, total cholesterol (TC), triglycerides (TG), low-density lipoprotein cholesterol (LDL-C), high-density lipoprotein cholesterol (HDL-C), and total cholesterol/HDL-C ratio were determined. Genotyping of rs1870377 and rs2071559 was performed by real-time PCR using Taqman probes.

Results: Two major dietary patterns were extracted from all subjects: 'Vegetables, fruits, and soy diet' (VFSD), and 'Rice, egg, and fish diet' (REFD). There were significant genetic associations for rs2071559 with TG $(p=0.048)$ in Malays, and in Chinese for rs 1870377 with TC $(p=0.001)$ and LDL-C ( $p=0.007)$, and rs2071559 on LDL-C $(p=0.048)$. The interaction effects of VEGFR-2 gene polymorphisms and dietary patterns were significant in Malays between rs2071559 and REFD on TG ( $p=0.005)$; and in Chinese between VFSD and rs1870377 on LDL-C ( $p=0.028)$ after adjusting for potential confounders. There were no significant genetic or dietary associations on blood lipids in Indians ( $p>0.05)$.
\end{abstract}

Conclusion: The significant associations and gene-diet interaction effects between VEGFR-2 gene polymorphisms and dietary patterns on blood lipids may pose hyperlipidemia and hypercholesterolemia risks in Malay and Chinese Malaysians.

Keywords: Gene-diet interaction, VEGFR-2 gene, Dietary pattern, Blood lipids, Malaysian adults.

\section{INTRODUCTION}

The global prevalence of chronic noncommunicable diseases (NCDs) such as cardiovascular disease (CVD) has been increasing in an alarming rate especially in the middle-and lowerincome countries. This is due to the rapid changes in globalization, urbanization, and lifestyle transitions $[1,2]$. Hence, in an upper middle-income country such as Malaysia, the top chronic NCDs are CVD, cancers, respiratory diseases, and diabetes mellitus [3]. Hypercholesterolemia which is major risk factor of CVD has become a major concern in the Malaysian population. Based on the National Health and Morbidity Survey 2011 (NHMS 2011), the latest reported prevalence of hypercholesterolemia among adults 18 years and older was approximately $33 \%$, and the prevalence has increased by almost double in just five years when compared to NHMS 2006 [4].

*Address correspondence to this author at the School of Biosciences, Taylor's University Lakeside campus, No. 1, Jalan Taylor's, 47500 Subang Jaya, Selangor, Malaysia; Tel: +603-5629-5090; Fax: +603-5629-5455;

E-mail: roselinewaikuan.yap@taylors.edu.my

E-ISSN: 1929-5634/14
Gene-environment and gene-diet interaction studies on chronic diseases are favored as these studies incorporate both non-modifiable (genetics) and modifiable risk factors (lifestyle habits). Several literatures have shown significant gene-diet interactions involving either single nutrient or dietary pattern on various risk factors of CVD such as metabolic syndrome [5,6,], obesity [7], and blood lipids [8]. In this study, vascular endothelial growth factor receptor-2 (VEGFR-2), also known as kinase insert domain receptor $(K D R)$ gene was selected as the candidate gene due to its major functions of vascular growth and repair [9] which is crucial in the cardiovascular system. Several studies have also reported significant associations of VEGFR-2 gene single nucleotide polymorphisms (SNPs) with several chronic NCDs. The missense SNP, rs1870377 of the VEGFR-2 gene was found to be associated with coronary heart disease (CHD) [10] while rs2071559 SNP, a regulatory SNP was associated with CHD [10], stroke [11], age-related macular degeneration (AMD) [12,13], and myocardial infarction [14].

In the Malaysian population, two studies reported associations between candidate gene polymorphisms 
and blood lipids in adults $[15,16]$, while only one study on dietary patterns [17]. Hence, gene-diet interaction studies involving candidate genes and dietary patterns on risk factors of CVD such as hyperlipidemia and hypercholesterolemia are scarce in the Malaysian population. To our knowledge no previous study has evaluated on the association of VEGFR-2 gene SNPS among the multi-ethnic Malaysian population. The investigation of such relationship and interaction could determine the susceptibility to increased risks of CVD. Hence, the aim of this study is to determine the association and gene-diet interaction effect between VEGFR-2 gene polymorphisms (rs1870377 and rs2071559) and dietary pattern on blood lipids in multiethnic Malaysian adults.

\section{MATERIALS AND METHODS}

\section{Study Design, Demographics, Health, Dietary Intake, and Lifestyle Information}

This study with convenience sampling approach recruited Malay, Chinese, and Indian adults residing in urban Klang Valley, Malaysia. The inclusion criteria include Malaysian citizenship, age 30-65 years old, not pregnant, and not hospitalized during the course of participation. The inclusion criterion of subjects must be of an offspring from two generations of the same ethnic group was also included to prevent a mix of genetic background present from inter-racial marriages among the ethnic groups. A standard questionnaire was used to obtain information pertaining to demographics, health, and lifestyle habits which were: age; gender; ethnic group; past or presence of common NCDs such as CVD, or on any prescribed medications for these diseases; smoking; alcohol consumption; and physical activity. Dietary intake information of all subjects was obtained using a semi-quantitative food frequency questionnaire (FFQ) previously described [18]. The questionnaires were presented in English for the Chinese and Indian subjects and were also translated to the Malay language for the Malay subjects. The questionnaires were either self-administered or were assisted by trained nutritionists for subjects who were illiterate. This study was approved by the Research Ethics Committee of University of Nagasaki, Japan. All subjects provided written informed consent.

\section{Anthropometric Measurements, Biomarkers, and Genotyping}

The height measurements of all subjects were measured to the nearest $0.1 \mathrm{~cm}$ using a height meter
(Seca body meter 208, Seca, Hamburg, Germany) and body mass index (BMI) values were obtained using a body fat analyzer (Omron HBF-356, Omron Health Care Co., Ltd., Kyoto, Japan). The systolic (SBP) and diastolic blood pressure (DBP) values were measured using an automated blood pressure monitor (Omron SEM-1, Omron Health Care Co., Ltd., Kyoto, Japan). The values of the following biomarkers were determined from each subject: glycated hemoglobin A1c (HbA1c); and blood lipids (total cholesterol, triglycerides, high-density lipoprotein cholesterol (HDLC), low-density lipoprotein cholesterol (LDL-C), and total cholesterol/HDL-C ratio) (UCSI Path Lab, Kuala Lumpur, Malaysia).

Buccal mucosal cells swabs were collected from all subjects using polyester fiber-tipped applicator swab (Falcon, Becton Dickinson and Company, Sparks, MD, USA) while DNA extraction and purification steps were performed using QIAamp DNA Blood Mini kit (Qiagen, Germantown, MD, USA). The real-time PCR system (ABI 7300, Applied Biosystems Japan, Tokyo, Japan and StepOne ${ }^{\mathrm{TM}}$, Applied Biosystems, Singapore) was applied for genotyping of VEGFR-2 gene polymorphisms (rs1870377 and rs2071559) of all of the subjects using Taqman ${ }^{\circledR}$ Genotyping Master Mix (Applied Biosystems, Foster City, CA, USA), Taqman $\AA$ GTXpress Master Mix (Applied Biosystems, Foster City, CA, USA) together with ready-made TaqMan probes based on reference SNP number, Taqman ${ }^{\circledR}$ SNP Genotyping Assays (Applied Biosystems, Foster City, CA, USA). All genotyping procedures were performed in reference to the manufacturer's protocol.

\section{Statistical Analysis}

The construction of dietary patterns using dietary intake information obtained from each subject was performed with factor analysis by applying principal component analysis. First, a primary analysis using the criterion of eigenvalues $>1.0$ was used to extract independent factors. The final number of independent factors which formed the total number of the major dietary patterns was then identified based on the Scree plot and the criterion to select factor loadings of values above 0.40 was used. Once major dietary patterns have been identified, factor scores were derived for each subject in each dietary pattern. Factor scores were then categorized into tertiles for further analysis involving association and gene-diet interaction.

The normality of each variable for continuous data was tested using Kolmogorov-Smirnov test. The 
association analyses were performed using a mixture of parametric tests and non-parametric tests: analysis of variance (ANOVA) with post-hoc analysis (Tukey test), analysis of covariance (ANCOVA) with post-hoc analysis (Sidak correction), and Kruskal-Wallis with post-hoc analysis (Dunnett's T3). Two-way ANOVA was used to determine gene-diet interactions. A probability value of $<0.05$ was considered statistically significant.

\section{RESULTS}

In this study, there were a total of 509 subjects comprising of 153 Malays ( $\mathrm{n}=39(25 \%)$ males and $\mathrm{n}=114 \quad(75 \%)$ females), 179 Chinese ( $\mathrm{n}=75 \quad(42 \%)$ males and $\mathrm{n}=104$ (58\%) females) and 177 Indians $(n=40(23 \%)$ males and $n=137(77 \%)$ females). Table 1 summarizes the anthropometric measurements, biomarkers, and genetic characteristics of the subjects by ethnic group. In terms of gender, there were more females compared to males with significant differences in mean for blood lipids. Females in all ethnic groups had significantly lower mean values for blood triglycerides, and total cholesterol/HDL-C ratio but higher in mean value for HDL-C compared to the males (data not shown, $p<0.05$ ). In comparison between ethnic groups for blood lipids, Malays had significantly higher mean values for total cholesterol and LDL-C compared to Chinese while Indians had significantly lower mean values for HDL-C compared to Malays and Chinese (Table 1).

Genetic characteristics have shown that the genotype frequencies among the Malays were: $24.2 \%$ of AA $(n=37), 45.8 \%$ of AT $(n=70)$ and $30.0 \%$ of TT $(n=46)$ for $r s 1870377$ and $15.7 \%$ of CC $(n=24), 51.0 \%$ of CT $(n=78)$ and $33.3 \%$ of TT $(n=51)$ for rs2071559; Chinese: $27.9 \%$ of AA ( $n=50), 48.6 \%$ of AT $(n=87$,$) and$ $23.5 \%$ of TT $(n=42)$ for $r s 1870377$ and $12.8 \%$ of CC

Table 1: Characteristics of Subjects

\begin{tabular}{|c|c|c|c|}
\hline & Malay $(n=153)$ & Chinese $(n=179)$ & Indian $(n=177)$ \\
\hline Age (years) & $41 \pm 7^{\mathrm{a}, \mathrm{b}}$ & $40 \pm 9^{b}$ & $43 \pm 9^{c}$ \\
\hline BMI $\left(\mathrm{kg} / \mathrm{m}^{2}\right)$ & $25.8 \pm 4.71^{a, c}$ & $24.2 \pm 4.95^{b}$ & $26.0 \pm 4.70^{c}$ \\
\hline Systolic blood pressure $(\mathrm{mmHg})$ & $121 \pm 15.6^{\mathrm{a}}$ & $122 \pm 14.9^{\mathrm{a}}$ & $123 \pm 15.8^{\mathrm{a}}$ \\
\hline Diastolic blood pressure $(\mathrm{mmHg})$ & $76.1 \pm 10.7^{\mathrm{a}}$ & $76.7 \pm 9.4^{\mathrm{a}}$ & $76.3 \pm 9.5^{\mathrm{a}}$ \\
\hline $\mathrm{HbA} 1 \mathrm{c}(\%$ of total $\mathrm{Hb})$ & $5.91 \pm 1.07^{\mathrm{a}, \mathrm{b}, \mathrm{c}}$ & $5.77 \pm 0.89^{b}$ & $6.23 \pm 1.44^{\mathrm{C}}$ \\
\hline Total cholesterol (mmol/L) & $5.65 \pm 0.99^{\mathrm{a}, \mathrm{c}}$ & $5.31 \pm 0.92^{b, c}$ & $5.41 \pm 0.94^{\mathrm{c}}$ \\
\hline Triglycerides (mmol/L) & $1.59 \pm 0.90^{\mathrm{a}}$ & $1.81 \pm 1.44^{\mathrm{a}}$ & $1.78 \pm 1.08^{\mathrm{a}}$ \\
\hline LDL-C (mmol/L) & $3.62 \pm 0.87^{\mathrm{a}, \mathrm{c}}$ & $3.08 \pm 0.87^{b}$ & $3.55 \pm 0.80^{c}$ \\
\hline HDL-C (mmol/L) & $1.35 \pm 0.36^{\mathrm{a}, \mathrm{b}}$ & $1.40 \pm 0.41^{b}$ & $1.19 \pm 0.31^{\mathrm{c}}$ \\
\hline Total cholesterol/HDL-C ratio & $4.50 \pm 1.53^{\mathrm{a}, \mathrm{b}, \mathrm{c}}$ & $4.11 \pm 1.46^{b}$ & $4.80 \pm 1.37^{c}$ \\
\hline rs 1870377 (A allele ; T allele) & $0.47 ; 0.53$ & $0.52 ; 0.48$ & $0.15 ; 0.85$ \\
\hline rs2071559 (C allele ; T allele) & $0.41 ; 0.59$ & $0.38 ; 0.62$ & $0.57 ; 0.43$ \\
\hline
\end{tabular}

Note: Data are presented in means \pm S.D.

${ }^{a, b, c}$ : Different letters indicate significant difference between two groups $(p<0.05)$.

Table 2: Association between Tertiles of Dietary Pattern 'Rice, Egg, and Fish Diet' and Blood Lipids in Malay Subjects

\begin{tabular}{|c|c|c|c|c|}
\hline Variables & $\begin{array}{c}\text { Tertile } 1 \\
(n=51)\end{array}$ & $\begin{array}{c}\text { Tertile } 2 \\
(n=51)\end{array}$ & $\begin{array}{c}\text { Tertile } 3 \\
(n=51)\end{array}$ & $p$-value \\
\hline Total cholesterol (mmol/L) & $5.49 \pm 0.85$ & $5.73 \pm 0.97$ & $5.73 \pm 1.14$ & 0.601 \\
\hline Triglycerides (mmol/L) & $1.32 \pm 0.64^{\mathrm{a}, \mathrm{b}}$ & $1.54 \pm 0.80^{\mathrm{b}, \mathrm{c}}$ & $1.91 \pm 1.11^{\mathrm{c}}$ & 0.015 \\
\hline LDL-C (mmol/L) & $3.39 \pm 0.74$ & $3.72 \pm 0.84$ & $3.72 \pm 0.98$ & 0.167 \\
\hline HDL-C (mmol/L) & $1.48 \pm 0.38^{a, b}$ & $1.34 \pm 0.36^{b, c}$ & $1.22 \pm 0.30^{c}$ & 0.001 \\
\hline Total cholesterol/HDL-C ratio & $3.89 \pm 1.02^{\mathrm{a}}$ & $4.57 \pm 1.49^{b, c}$ & $5.03 \pm 1.78^{c}$ & 0.001 \\
\hline
\end{tabular}

Note: Data are presented in means \pm S.D.

a,b,c: Different letters indicate significant difference between two groups $(p<0.05)$. 
$(n=23), 50.3 \%$ of CT $(n=90)$ and $36.9 \%$ of TT $(n=66)$ for rs2071559; and Indians: $1.7 \%$ of AA ( $n=3), 27.7 \%$ of AT $(n=49)$ and $70.6 \%$ of TT $(n=125)$ for $r s 1870377$ and $32.2 \%$ of CC $(n=57), 48.6 \%$ of CT $(n=86)$ and $19.2 \%$ of TT $(n=34)$ for rs2071559. The genotypes at both VEGFR-2 gene SNP sites were conformed to the Hardy-Weinberg equilibrium using a web-based tool [19]. The minor allele frequencies for VEGFR-2 gene SNPs (rs1870377 and rs2071559) by ethnic groups were more than 0.2 except for rs 1870337 SNP among Indians.

Two major dietary patterns were extracted from all 509 subjects in this study: 'Vegetables, fruits, and soy diet' (VFSD), and 'Rice, egg, and fish diet' (REFD). Dietary pattern of VFSD was derived from high consumption of various types of vegetables (green leafy vegetables, cabbage, cauliflower, Chinese cabbage, and broccoli), fruits (papaya, mango, pineapple, starfruit, and papaya), and soybean curd while dietary pattern of REFD was comprised of rice, chicken egg, and fish. The dietary patterns explained $28.9 \%$ of the variance in the usual dietary intakes of the subjects. Table 2 shows the mean values of blood lipids by tertile for REFD for Malay subjects whereby significant associations were obtained for blood lipids triglycerides, HDL-C, and total cholesterol/HDL-C ratio. Malay subjects in Tertile 3 of REFD had poorer blood lipid status compared to subjects in Tertile 1 with significantly higher in mean values for triglycerides $(p=0.005)$, total cholesterol/HDL-C ratio $(p<0.001)$, and significantly lower in mean values for HDL-C $(p<0.001)$. Further analysis with the adjustment of confounding variables namely age, smoking, and physical activity was performed for HDL-C due to its normal distribution of data and similar finding $(p=0.006)$ was obtained (data not shown). Significant associations for blood lipids were not obtained for VFSD in Malay subjects, and also for both dietary patterns in Chinese and Indian subjects (data not shown).

The genetic associations between VEGFR-2 gene SNPs (rs1870377 and rs2017559) and blood lipids were also determined in this study. The present study showed significant associations only in Malays and Chinese. In the Malay group, CC-homozygote subjects of rs2071559 had significantly higher mean in blood triglyceride levels $(1.96 \pm 0.25 \mathrm{mmol} / \mathrm{L})$ compared to CT-

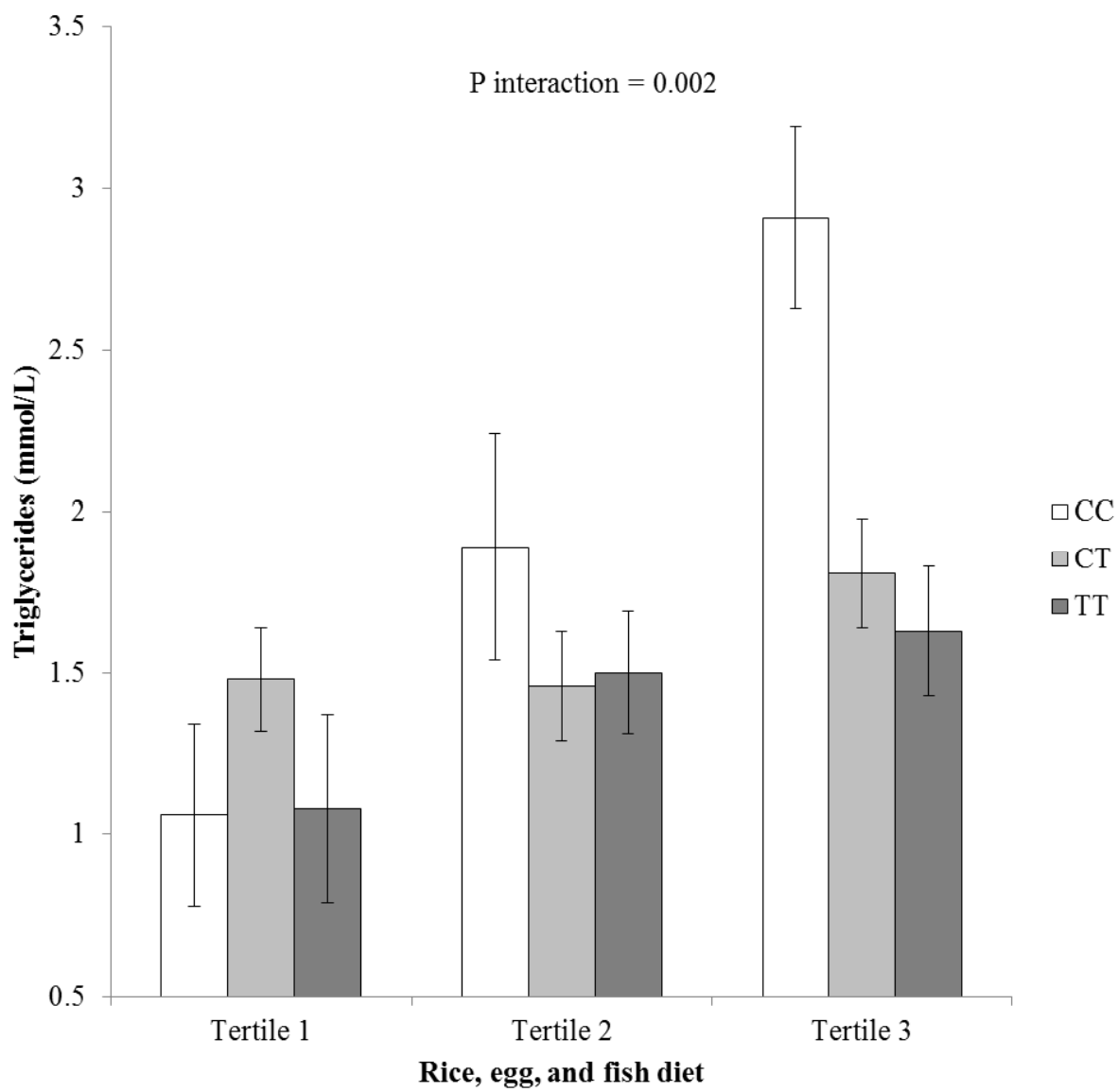

Figure 1: Interaction between tertiles of 'Rice, egg, and fish diet' and genotypes of rs2071559 (CC, CT, and TT) on blood triglyceride $(\mathrm{mmol} / \mathrm{L})$ in Malay subjects. Values are in mean $\pm S E$ adjusted for age, smoking, and physical activity. 
homozygote $(1.58 \pm 0.06 \mathrm{mmol} / \mathrm{L})$ and TT-homozygote subjects $(1.43 \pm 0.12 \mathrm{mmol} / \mathrm{L})(p=0.017)$. The significant associations involving both rs1870377 and rs2071559 SNPs on blood lipids among Chinese subjects were described previously in which TT-homozygote subjects had higher risks for LDL-C and/or total cholesterol [18]. No significant associations were obtained between genotypes of VEGFR-2 gene SNPs (rs1870377 and rs2071559) and blood lipids in Indians (data not shown).

Gene-diet interactions effects between VEGFR-2 gene SNPs (rs1870377 and rs2071559) and dietary patterns (VFSD and REFD) on blood lipids by ethnic group were determined in this study and only significant gene-diet interaction effects were obtained in Malays and Chinese after adjusting for potential confounding factors age, smoking, physical activity, and alcohol consumption. In Malay subjects, there was significant gene-diet interaction effect between VEGFR-2 gene rs2017559 SNP and dietary pattern, REFD on blood triglycerides (Figure 1). It was observed that for the combination by genotype of rs2071559 and tertile 3 of REFD (high consumption of rice, egg, and fish diet), CC-homozygote subjects $(n=9)$ had the highest mean in blood triglycerides followed by CT-heterozygote subjects $(n=24)$, and TT-homozygote subjects $(n=18)$. The combination of CC-homozygote and tertile 3 of REFD also had the highest mean in blood triglycerides compared to all other combinations. Significant gene-diet interaction effect was also obtained in Chinese subjects between VEGFR-2 gene rs1870377 SNP and dietary pattern, VFSD on LDL-C levels (Figure 2). It is shown that the combination by genotype of rs 1870377 and tertile 1 (low consumption of vegetables, fruits, soy diet), AA-homozygote subjects $(n=23)$ had the highest mean in LDL-C levels followed by AT-heterozygote subjects $(n=25)$, and TThomozygote subjects $(n=23)$. However, a reverse order was obtained in tertile 2 of VFSD, and in tertile 3 , TThomozygote $(n=13)$ had the highest mean in LDL-C levels compared to all other combinations. No significant gene-diet interaction effects were observed

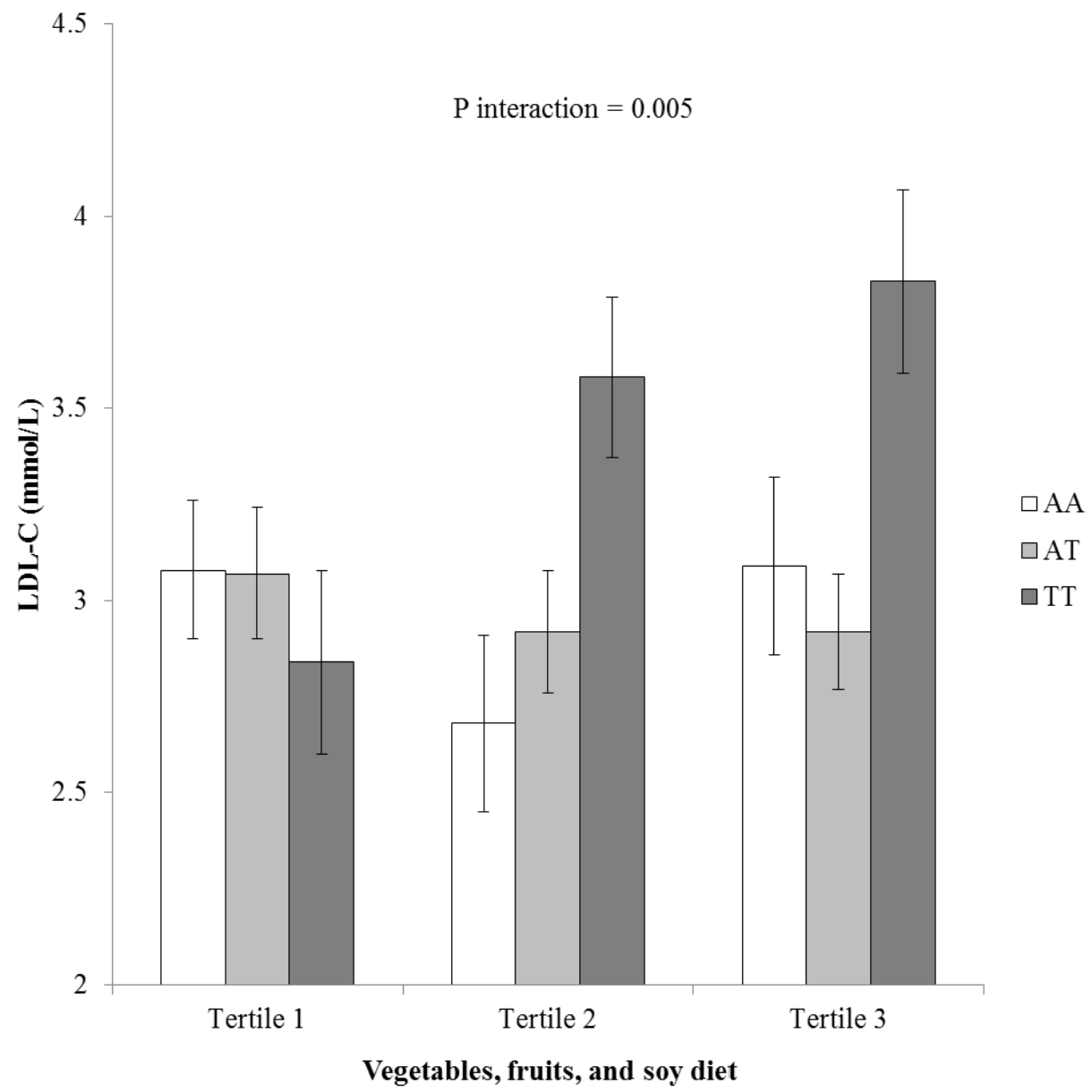

Figure 2: Interaction between tertiles of 'Vegetables, fruits, and soy diet' and genotypes of rs1870377 (AA, AT, and TT) on blood LDL-C (mmol/L) in Chinese subjects. Values are in mean \pm SE adjusted for age, smoking, physical activity, and alcohol consumption. 
between VEGFR-2 gene SNPs and dietary patterns on blood lipids among Indians (data not shown).

\section{DISCUSSION}

In this study, the association and gene-diet interaction effect between VEGFR-2 gene polymorphisms (rs1870377 and rs2071559) and dietary pattern on blood lipids were determined in the three main ethnic groups of Malaysian adults. The findings of this study revealed that associations and gene-diet interactions differed among the ethnic groups and significant findings were obtained in all ethnic groups except for the Indians. In the Malay subjects, significant associations were obtained for dietary pattern of REFD, rs2071559 SNP, and gene-diet interaction (rs2071559 and REFD) particularly on blood triglycerides. On the other hand, in the Chinese subjects significant associations were obtained for both rs 1870377 and rs2071559 SNPs, and gene-diet interaction (rs1870377 and VFSD) particularly on LDL-C.

In terms of blood lipids status, the present study showed that Malay and Indian subjects had higher risk of blood lipids compared to the Chinese subjects. Our findings are consistent with the latest NHMS 2011 which revealed that prevalence of hypercholesterolemia was highest in the Malays followed by Indians and Chinese [4]. There is limited information on dietary patterns of the Malaysian population. A study which investigated on the association of dietary patterns with oral cancer risk revealed significant increased risk in two dietary patterns, one which is high in beverages and starches, and the other which is high in dairy, fermented/salted and meat/by-products [20]. A recent study on Malaysian adolescents aged 12-13 years old has indicated significant association between high-energy food pattern with general cognitive ability [17]. In this study, two major dietary patterns (VFSD and REFD) were extracted from multi-ethnic Malaysian subjects. However, it was only shown that Malay subjects in the highest tertile of dietary pattern REFD i.e. high consumption of rice, egg, and fish had significant higher risk of several blood lipids.

The associations between VEGFR-2 gene polymorphisms (rs1870377 and rs2071559) and blood lipids were found to be significant only among Malay and Chinese subjects of this study. Among the Malaysian subjects, C allele of rs2071559 in Malay subjects had significantly higher mean in blood triglycerides levels while T allele of both rs 1870377 and rs2071559 in Chinese subjects had significantly higher risk of blood cholesterol levels. Previous study involving Japanese subjects reported no significant associations between VEGFR-2 gene SNPs (rs1870377 and rs2071559) on the development of coronary artery lesion in Kawasaki disease subjects [21]. There were two studies involving the Han Chinese population which reported that the $A$ allele of rs 1870377 and $C$ allele of rs2071559 had a higher risk of CHD while $C$ allele of rs2071559 had a lower risk in susceptibility to stroke and recurrence $[10,11]$. Another study among Caucasians originated from Italy has reported that T allele of rs2071559 had higher risk of AMD [12] while another study also conducted in Italy has reported that the number of TT-homozygote subjects were lower among patients with neovascular AMD [13]. A recent study on Caucasians of Slavin origin indicated that CC genotype of rs2071559 had higher risk of myocardial infarction in type 2 diabetes mellitus patients [14]. Based on the literatures on the related VEGFR-2 gene SNPs, it can be summarized that there is insufficient data on rs1870377 SNP while there are more evidence demonstrating that $\mathrm{C}$ allele of rs2071559 may increase the risk of CVDs, which is consistent with our finding among the Malay subjects. However, the differences may also indicate that these two SNPs could have different effects on selected populations and ethnic groups in a wide range of chronic diseases/conditions.

The present study showed significant gene-diet interaction effects on blood triglycerides in Malay subjects and LDL-C in Chinese subjects. It was observed in the Malay subjects that the gene-diet interaction between CC-homozygote of rs2071559 and tertile 3 of REFD had higher mean blood triglycerides levels than individual associations of rs2071559 SNP by genotype and dietary pattern of REFD by tertiles. The interaction between the dietary component and regulatory SNP, rs2071559 may influence the expression levels of VEGFR-2 which resulted to the increased levels of blood triglycerides. It was reported that CC genotype of rs2071559 was associated with reduced expression of VEGFR-2 in Han Chinese subjects [10] while a recent study showed significantly higher fasting serum VEGF levels in type II diabetic mellitus patients without coronary artery disease when compared with other genotypes [14]. On the other hand, in Chinese subjects, significant dietary pattern associations were not obtained for blood lipids, therefore the dietary pattern of VFSD could enhance polymorphism effects of rs 1870377 by influencing the 
protein activity of VEGFR-2 contributing to the significant gene-diet interactions. In addition, VEGFR-2 gene is also expressed via several types of cells including endothelial progenitor cells (EPCs) [9] and several studies have reported significant associations between VEGFR-2 activity indicated by number of EPCs with several chronic diseases. It was reported that the number of circulation EPCs was found to be low in patients with hypercholesterolemia [22,23]

In this study, we did not obtain any significant associations or gene-interaction effects in the Indian subjects even though increased risk of blood lipids was observed when compared with Chinese subjects. In terms of dietary patterns associations, the possible reason could be due to food preparation methods which were not determined in this study. Food preparation or cooking methods such as the use of lard, and deep fat frying may affect the quality of the diet (e.g. high in total saturated fats or trans fatty acids). It is reported in a study that the increasing prevalence of metabolic syndrome among Asians Indians could be due to a dietary pattern of high consumption of saturated fat [24]. As for the insignificant genetic associations, the possible reason could be due to the small MAF of less than 0.2 for VEGFR-2 gene rs1870377 SNP with only three AAhomozygote subjects among the Indian subjects. It was also observed that there was a distinct difference in allele frequencies between the Indian subjects compared to Malay, and Chinese subjects. The Indians were more dominant in the $\mathrm{C}$ allele which may not contribute to any effect on the expressions levels of VEGFR-2 gene.

The limitations of this study should be noted, in which the sample size of the subjects was limited in each ethnic group, and there were more females compared to males. A larger sample size may help to strengthen the statistical power of the study. The FFQ is a reliable tool used in obtaining dietary intake information, however due to its retrospective nature of this tool, data on the food intake obtained is relied on the information provided by the participants, and may not reflect the actual intake. Lastly, the cross-sectional study design of this study limits the interpretations to associations, and gene-diet interactions. Any interpretations on the causal effects of VEGFR-2 gene polymorphisms and dietary patterns on blood lipids could not be obtained in this study. This may be the first study involving the associations and gene-diet interactions between VEGFR-2 gene polymorphisms and dietary patterns among Malaysian adults hence further studies involving a larger sample size should be conducted to confirm the findings of this study. In addition, similar investigations can also be performed in other candidate genes associated with blood lipids.

In summary, in the Malay subjects the dietary pattern of REFD was associated with blood lipids, C allele of rs2071559, and gene-diet interaction effect with the combination of CC and tertile 3 of REFD had increased risk of blood triglycerides. In the Chinese subjects, $\mathrm{T}$ allele of both VEGFR-2 gene SNPs (rs1870377 and rs2071559), and gene-diet interaction effect with the combination TT and tertile 3 of VFSD had increased risk of LDL-C. Hence, significant associations and gene-diet interaction effects between VEGFR-2 gene polymorphisms and dietary patterns on blood lipids obtained in this study may provide insights on the hyperlipidemia and hypercholesterolemia risks in Malay and Chinese Malaysians. With that, investigations on gene-diet interactions studies are required to achieve the goal of personalized nutrition in the future.

\section{ACKNOWLEDGEMENT}

The authors are very grateful to all of the study participants for their time and cooperation.

\section{REFERENCES}

[1] Narayan KM, Ali MK, Koplan JP. Global noncommunicable diseases-where worlds meet. N Engl J Med 2010; 363: 1196-1198.

http://dx.doi.org/10.1056/NEJMp1002024

[2] World Health Organization (WHO) Global status report: noncommunicable diseases 2010: NCDs and development. Geneva, WHO, Switzerland 2011.

[3] World Health Organization (WHO). Noncommunicable diseases country profiles 2011: Malaysia. Geneva, WHO, Switzerland 2011.

[4] Institute for Public Health (IPH). National Healthy and Morbidity Survey 2011 (NHMS 2011) Vol. II: Non Communicable Disease 2011.

[5] Phillips CM, Tieryney AC, Roche HM. Gene-nutrient interactions in the metabolic syndrome. J Nutrigenet Nutrigenomics 2008; 1: 136-151. http://dx.doi.org/10.1159/000112461

[6] Baylin A, Deka R, Tuitele J, Viali S, Weeks DE, McGarvey ST. INSIG2 variants, dietary patterns and metabolic risk in Samoa. Eur J Clin Nutr 2013; 67: 101-107. http://dx.doi.org/10.1038/ejcn.2012.124

[7] Anderson AL, Harris TB, Houston DK, Tlavksy FA, Lee JS, Sellmeyer DE, et al. Relationships of dietary patterns with body composition in older adults differ by gender and PPAR$\gamma$ Pro12Ala genotype. Eur J Nutr 2010; 49: 385-394. http://dx.doi.org/10.1007/s00394-010-0096-9

[8] Rudkowska I, Dewailly E, Hegele RA, Boiteau V, DubeLinteau A, Abdous B, et al. Gene-diet interactions on plasma lipid levels in the Inuit population. Br J Nutr 2013; 108: 953961.

http://dx.doi.org/10.1017/S0007114512002231 
[9] Holmes K, Roberts OL, Thomas AM, Cross MJ. Vascular endothelial growth factor receptor-2: structure, function, intracellular signaling and therapeutic inhibition. Cell Signal 2007; 19: 2003-2012.

http://dx.doi.org/10.1016/j.cellsig.2007.05.013

[10] Wang Y, Zheng Y, Zhang W, Yu Y, Lou K, Zhang Y, et al. Polymorphisms of KDR gene are associated with coronary heart disease. J Am Coll Cardiol 2007; 50: 760-767. http://dx.doi.org/10.1016/j.jacc.2007.04.074

[11] Zhang W, Sun K, Zhen Y, Wang D, Wang Y, Chen J, et al. VEGF Receptor-2 variants are associated with susceptibility to stroke and recurrence. Stroke 2009; 40: 2720-2726. http://dx.doi.org/10.1161/STROKEAHA.109.554394

[12] Galan A, Ferlin A, Caretti L, Buson G, Sato G, Frigo AC, et al. Association of age-related macular degeneration with polymorphisms in vascular endothelial growth factor and its receptor. Ophthalmology 2010; 117: 1769-1774. http://dx.doi.org/10.1016/i.ophtha.2010.01.030

[13] Lazzeri $S$, Orlandi $P$, Fugis $M$, Fiorravanti $A$, Cascio $E$, Desidero TD, et al. The rs2071559 AA VEGFR-2 genotype frequency is significantly lower in neovascular age-related macular degeneration patients. Scientific World Journal 2012; 420190. Available from: http://www.ncbi.nlm.nih. gov/pmc/articles/PMC3415178/

[14] Kariž S, Petrovič D. Minor association of kinase insert domain-containing receptor gene polymorphism (rs2071559) with myocardial infarction in Caucasians with type 2 diabetes mellitus: case-control cross-sectional study. Clin Biochem 2014; 47: 192-196. http://dx.doi.org/10.1016/j.clinbiochem.2014.08.004

[15] Apalasamy YD, Ming MF, Rampal S, Bulgiba A, Mohamed Z. Genetic association of SNPS in the FTO gene and predisposition to obesity in Malaysian Malays. Braz $\mathrm{J}$ Med Biol Res 2012; 45: 1119-26.

http://dx.doi.org/10.1590/S0100-879X2012007500134

[16] Apalasamy YD, Ming MF, Rampal S, Bulgiba A, Mohamed Z. Gender-dependent association of a $\beta_{2}$-adrenergic gene variant with obesity parameters in Malaysian Malays. Asia Pac J Public Health 2011; 1: 1-12. Available from: http://aph.sagepub.com/content/early/2011/12/08/101053951 1430250.long
[17] Nurliyana AR, Mohd Nasir MT, Zalilah MS, Rohani A. Dietary patterns and cognitive ability among 12- to 13 year-old adolescents in Selangor, Malaysia. Public Health Nutr 2014; 10: $1-10$.

http://dx.doi.org/10.1017/S1368980014000068

[18] Yap RWK, Shidoji Y, Hon WM, Masaki M. Interaction between VEGF receptor-2 (VEGFR-2) gene polymorphisms and dietary patterns on blood glucose and lipid levels in Chinese Malaysian adults. J Nutrigenet Nutrigenomics 2011; 4: 309-321. http://dx.doi.org/10.1159/000334358

[19] Rodriguez S, Gaunt TR, Day INM. Hardy-Weinberg equilibrium testing of biological ascertainment for Mendelian randomization studies. Am J Epidemiol 2009; 169(4): 505514. http://dx.doi.org/10.1093/aje/kwn359

[20] Helen-Ng LC, Razak IA, Ghani WM, Marhazlinda J, Norain AT, Raja Jallaludin RL, et al. Dietary pattern and oral cancer risk-a factor analysis study. Community Dent Oral Epidemiol 2012; 40(6): 560-566.

http://dx.doi.org/10.1111/j.1600-0528.2012.00704.x

[21] Kariyazono $\mathrm{H}$, Ohno $\mathrm{T}$, Khajoee $\mathrm{V}$, Ihara K, Kusuhara K, Kinukawa N, et al. Association of vascular endothelial growth factor (VEGF) and VEGF receptor gene polymorphisms with coronary artery lesions of Kawasaki disease. Pediatr Res 2004; 156: 953-959.

http://dx.doi.org/10.1203/01.PDR.0000145280.26284.B9

[22] Chen JZ, Zhang FR, Tao QM, Wang XX, Zhu JH, Zhu JH. Number and activity of endothelial progenitor cells from peripheral blood in patients with hypercholesterolemia. Clin Sci (Lond) 2004; 107: 273-280. http://dx.doi.org/10.1042/CS20030389

[23] Ramunni A, Brescia P, Dambra P, Capuzzimati L, Ria R, De Tullio $\mathrm{G}$, et al. Effect of low-density lipoprotein apheresis on circulating endothelial progenitor cells in familial hypercholesterolemia. Blood Purif 2010; 29: 383-389. http://dx.doi.org/10.1159/000314650

[24] Das M, Pal S, Arnab G. Association of metabolic syndrome with obesity measures, metabolic profiles, and intake of dietary fatty acids in people of Asian Indian origin. $J$ Cardiovasc Dis Res 2010; 1(3): 130-135. http://dx.doi.org/10.4103/0975-3583.70911 